\title{
A Unified Algebraic Technique for Eigenvalues and Eigenvectors in Quaternionic and Split Quaternionic Mechanics
}

\author{
Zhenwei Guo', Gang Wang1, Dong Zhang'2, Tongsong Jiang1,2,3* \\ ${ }^{1}$ School of Mathematical Science, Liaocheng University, Liaocheng, China \\ ${ }^{2}$ College of Mathematics and Systems Science, Shandong University of Science and Technology, Qingdao, China \\ ${ }^{3}$ School of Mathematics and Statistics, Heze University, Heze, China \\ Email: guozhenweilcu@163.com,dz_zhangdong@sina.com, *jiangtongsong@sina.com
}

How to cite this paper: Guo, Z.W., Wang, G., Zhang, D. and Jiang, T.S. (2019) A Unified Algebraic Technique for Eigenvalues and Eigenvectors in Quaternionic and Split Quaternionic Mechanics. Journal of Applied Mathematics and Physics, 7, 1929-1940. https://doi.org/10.4236/jamp.2019.79133

Received: July 28, 2019

Accepted: August 26, 2019

Published: August 29, 2019

Copyright $\odot 2019$ by author(s) and Scientific Research Publishing Inc. This work is licensed under the Creative Commons Attribution International License (CC BY 4.0).

http://creativecommons.org/licenses/by/4.0/

\begin{abstract}
This paper aims to present, in a unified manner, the algebraic techniques of eigen-problem which are valid on both the quaternions and split quaternions. This paper studies eigenvalues and eigenvectors of the v-quaternion matrices by means of the complex representation of the v-quaternion matrices, and derives an algebraic technique to find the eigenvalues and eigenvectors of v-quaternion matrices. This paper also gives a unification of algebraic techniques for eigenvalues and eigenvectors in quaternionic and split quaternionic mechanics.
\end{abstract}

\section{Keywords}

v-Quaternion, Complex Representation, Quaternion, Split Quaternion, Eigenvalues, Eigenvectors

\section{Introduction}

A quaternion, which was found in 1840 by William Rowan Hamilton [1], is in the form of $q=q_{1}+q_{2} \mathrm{i}+q_{3} \mathrm{j}+q_{4} \mathrm{k}, \quad \mathrm{i}^{2}=\mathrm{j}^{2}=\mathrm{k}^{2}=-1, \quad \mathrm{ijk}=-1$, in which $q_{1}, q_{2}, q_{3}, q_{4} \in \mathbf{R}$, and $\mathrm{ij}=-\mathrm{ji}=\mathrm{k}, \mathrm{jk}=-\mathrm{kj}=\mathrm{i}, \mathrm{ki}=-\mathrm{ik}=\mathrm{j}$. A split quaternion, which was found in 1849 by James Cockle [2], is in the form of $q=q_{1}+q_{2} \mathrm{i}+q_{3} \mathrm{j}+q_{4} \mathrm{k} \quad, \quad \mathrm{i}^{2}=-1 \quad, \quad \mathrm{j}^{2}=\mathrm{k}^{2}=1 \quad, \quad \mathrm{ijk}=1 \quad, \quad$ in $\quad$ which $q_{1}, q_{2}, q_{3}, q_{4} \in \mathbf{R}$, and $\mathrm{ij}=-\mathrm{ji}=\mathrm{k}, \mathrm{jk}=-\mathrm{kj}=-\mathrm{i}, \mathrm{ki}=-\mathrm{ik}=\mathrm{j}$. Denote the sets of quaternions and split quaternions by $\mathbf{H}$ and $\mathbf{H}_{s}$, respectively. They are all associative and non-commutative 4-dimensional Clifford algebra, but the $\mathbf{H}_{s}$ is a more complex algebraic structure, because the $\mathbf{H}_{s}$ contains zero divisors, 
nilpotent elements and nontrivial idempotents.

Quaternion and split quaternion are playing an increasingly important role in many fields. Till now, quaternions are widely used in programming video games, controlling spacecrafts, computer graphics, control theory, signal processing, quantum physics [3] [4] [5]. And there are many applications of split quaternions [6] [7] [8] [9], split quaternion is one of the tools for studying modern quantum mechanics in the physical, and Lorentzian rotations can be represented by split quaternions. Eigenvalue and eigenvector problems of quaternion and split quaternion matrices have many applications and are the basic tools of many scientific researches, therefore they have strong research significance. For quaternion, in [10], the author studied the problems of eigenvalues and eigenvectors of quaternion matrices by means of complex representation and companion vector. In [11], the author applied the Lefschetz Fixed Point Theorem to show that every square matrix over the quaternions has right eigenvalues, in addition, the author classified these eigenvalues and discussed some of their properties. For split quaternion, in [12], by means of complex representation of a split quaternion matrix, the authors studied the problems of right split quaternion eigenvalues and eigenvectors of a split quaternion matrix. In [13], the authors discussed the properties of complex eigenvalues of a split quaternion matrix, and gave an extension of Gershgorin theorem.

Because there are amazing relationships between quaternions and split quaternions [14] [15], the purpose of this paper is to unify and generalize them to the general case, then use a common method to find their eigenvalues and eigenvectors. Therefore, a new algebraic structure is defined, it is v-quaternion.

A v-quaternion is in the form of

$$
q=q_{1}+q_{2} \mathrm{i}+q_{3} \mathrm{j}+q_{4} \mathrm{k}, \mathrm{i}^{2}=-1, \mathrm{j}^{2}=\mathrm{k}^{2}=\mathrm{ijk}=v,
$$

in which $0 \neq v \in \mathbf{R}, \quad q_{1}, q_{2}, q_{3}, q_{4} \in \mathbf{R}, \quad$ and $\mathrm{ij}=-\mathrm{ji}=\mathrm{k}, \quad \mathrm{jk}=-\mathrm{kj}=-v \mathrm{i}$, $\mathrm{ik}=-\mathrm{ki}=-\mathrm{j}$. Similarly, denote the sets of $\mathrm{v}$-quaternions by $\mathbf{H}_{v}$. Obviously, the set of all v-quaternion is also a non-commutative 4-dimensional Clifford algebra. Specially, when $v=-1$, the ring of the v-quaternion $\mathbf{H}_{v}$ is the ring of the quaternion $\mathbf{H}$; when $v=1$, the ring of the v-quaternion $\mathbf{H}_{v}$ is the ring of the split quaternion $\mathbf{H}_{s}$.

Before start this paper, first introduce the necessary symbols and preliminary knowledge. Let $\mathbf{R}$ be the real number field, $\mathbf{C}=\mathbf{R} \oplus \mathbf{R i}$ the complex number field, and $\mathbf{H}_{v}=\mathbf{R} \oplus \mathbf{R i} \oplus \mathbf{R} \mathbf{j} \oplus \mathbf{R} \mathbf{k}$ the v-quaternion ring, in which $\mathrm{i}^{2}=-1, \mathrm{j}^{2}=v, \mathrm{k}^{2}=v, \quad \mathrm{ij}=-\mathrm{ji}=\mathrm{k}, \quad \mathrm{jk}=-\mathrm{kj}=-v \mathrm{i}, \quad \mathrm{ik}=-\mathrm{ki}=-\mathrm{j}$. If $q=q_{1}+q_{2} \mathrm{i}+q_{3} \mathrm{j}+q_{4} \mathrm{k} \in \mathbf{H}_{v}, \quad \bar{q}=q_{1}-q_{2} \mathrm{i}-q_{3} \mathrm{j}-q_{4} \mathrm{k}$ is the conjugate of $q$, then $q \bar{q}=q_{1}^{2}+q_{2}^{2}-v q_{3}^{2}-v q_{4}^{2}$, the norm of $q$ is defined to be $|q|=\sqrt{|q \bar{q}|}=\sqrt{\left|q_{1}^{2}+q_{2}^{2}-v q_{3}^{2}-v q_{4}^{2}\right|}$. For any matrix $A=\left(a_{s t}\right) \in \mathbf{H}_{v}^{m \times n}, \bar{A}=\left(\bar{a}_{s t}\right)$, $A^{\mathrm{T}}=\left(a_{t s}\right), A^{*}=\left(\bar{a}_{t s}\right)$ denote the conjugate, the transpose, the conjugate transpose of the matrix $A$, respectively. A v-quaternion matrix $A \in \mathbf{H}_{v}^{n \times n}\left(a \in \mathbf{H}_{v}\right)$ is said to be nonsingular if there exists a v-quaternion matrix $B \in \mathbf{H}_{v}^{n \times n}\left(b \in \mathbf{H}_{v}\right)$ 
such that $A B=B A=I_{n}(a b=b a=1)$. Two $n \times n$ v-quaternion matrices $A$ and $B$ are said to be similar if there exists a nonsingular v-quaternion matrix $P$ such that $P^{-1} A P=B$, this is written as $A \sim B$. For $A \in \mathbf{C}^{m \times m}, f_{A}(\lambda)$ denotes the characteristic polynomial of $A$.

In this paper, we study the problem of right eigenvalues and associated right eigenvectors of the v-quaternion matrix. A v-quaternion $\lambda$ is said to be a right eigenvalue provided that $A \alpha=\alpha \lambda$ for nonzero vector $\alpha$, and $\alpha$ is said to be an eigenvector related to the right eigenvalue $\lambda$.

\section{Equivalence Classes of v-Quaternions}

Two v-quaternions $p$ and $q$ are said to be similar if there exists a nonsingular v-quaternion $x$ such that $x^{-1} p x=q$, written as $p \sim q$. It is easy to find that $p$ and $q$ are similar if and only if a unit v-quaternion $u$ such that $u^{-1} p u=q$, then two similar v-quaternions have the same norm. Obviously, $\sim$ is an equivalence relation on the $v$-quaternions. Denote the equivalence classes containing the v-quaternion $q$ by $[q]$.

It is easy to prove the following result by direct calculation.

Proposition 2.1 If $q=q_{1}+q_{2} \mathrm{i}+q_{3} \mathrm{j}+q_{4} \mathrm{k} \quad$ is a v-quaternion, then

1) If $v\left(q_{3}^{2}+q_{4}^{2}\right)-q_{2}^{2}<0$, then construct a nonsingular v-quaternion $x$ such that

$$
\left.x^{-1} q x=q_{1}+\sqrt{q_{2}^{2}-v\left(q_{3}^{2}+q_{4}^{2}\right)}\right),
$$

in which $x_{1}=\epsilon \sqrt{v \epsilon} q_{3}+\left(\sqrt{q_{2}^{2}-v\left(q_{3}^{2}+q_{4}^{2}\right)}+q_{2}+\epsilon \sqrt{v \epsilon} q_{4}\right) \mathrm{i}+q_{3} \mathrm{j}$

$$
+\left(q_{4}-\sqrt{\frac{q_{2}^{2}-v\left(q_{3}^{2}+q_{4}^{2}\right)}{v \epsilon}}+\frac{q_{2}}{\sqrt{v \epsilon}}\right) \mathrm{k} .
$$

2) If $v\left(q_{3}^{2}+q_{4}^{2}\right)-q_{2}^{2}>0$, there must be $v>0$ at this point, then construct a nonsingular v-quaternion $x$ such that

$$
x^{-1} q x=q_{1}+\sqrt{q_{3}^{2}+q_{4}^{2}-\frac{1}{v} q_{2}^{2}} \mathrm{j},
$$

in which $x=\sqrt{v} q_{3}+\sqrt{v\left(q_{3}^{2}+q_{4}^{2}\right)-q_{2}^{2}}-\left(q_{2}-\sqrt{v} q_{4}\right) \mathrm{i}$

$$
-\left(q_{3}+\sqrt{q_{3}^{2}+q_{4}^{2}-\frac{1}{v} q_{2}^{2}}\right) \mathrm{j}+\left(\frac{1}{\sqrt{v}} q_{2}-q_{4}\right) \mathrm{k} .
$$

3) If $q_{2}^{2}=v\left(q_{3}^{2}+q_{4}^{2}\right)$, when $q_{2}=0$, then $q=q_{1}$, and

$$
x^{-1} q x=q_{1},
$$

in which $x=1$. When $q_{2} \neq 0$, and this case also implies $v>0$, then construct a nonsingular v-quaternion $x$ such that

$$
x^{-1} q x=q_{1}+\mathrm{i}+\frac{1}{\sqrt{v}} \mathrm{j},
$$

in which $x=1+\sqrt{v} q_{3}+\sqrt{v} q_{4} \mathrm{i}+\frac{1}{\sqrt{v}}\left(q_{2}-1\right) \mathrm{k}$. 
The following two special cases about quaternion and split quaternion comes from Proposition 2.1 with $v=-1$ and $v=1$, respectively.

Corollary 2.2 If $q=q_{1}+q_{2} \mathrm{i}+q_{3} \mathrm{j}+q_{4} \mathrm{k}$ is a quaternion, then construct a nonsingular quaternion $x$ such that

$$
x^{-1} q x=q_{1}+\sqrt{q_{2}^{2}+q_{3}^{2}+q_{4}^{2}} \text { i, }
$$

in which $x=-q_{3}+\left(q_{2}-q_{4}+\sqrt{q_{2}^{2}+q_{3}^{2}+q_{4}^{2}}\right) \mathrm{i}+q_{3} \mathrm{j}+\left(q_{2}+q_{4}-\sqrt{q_{2}^{2}+q_{3}^{2}+q_{4}^{2}}\right) \mathrm{k}$.

Corollary 2.3 If $q=q_{1}+q_{2} \mathrm{i}+q_{3} \mathrm{j}+q_{4} \mathrm{k}$ is a split quaternion, then

1) If $q_{2}^{2}>q_{3}^{2}+q_{4}^{2}$, then construct a nonsingular split quaternion $x$ such that

$$
x^{-1} q x=q_{1}+\sqrt{q_{2}^{2}-q_{3}^{2}-q_{4}^{2}} \mathrm{i}
$$

in which $x=q_{3}+\left(q_{2}+q_{4}+\sqrt{q_{2}^{2}-q_{3}^{2}-q_{4}^{2}}\right) \mathrm{i}+q_{3} \mathrm{j}+\left(q_{2}+q_{4}-\sqrt{q_{2}^{2}-q_{3}^{2}-q_{4}^{2}}\right) \mathrm{k}$.

2) If $q_{2}^{2}<q_{3}^{2}+q_{4}^{2}$, then construct a nonsingular split quaternion $x$ such that

$$
x^{-1} q x=q_{1}+\sqrt{q_{3}^{2}+q_{4}^{2}-q_{2}^{2}} \mathrm{j},
$$

in which $x=q_{3}+\sqrt{q_{3}^{2}+q_{4}^{2}-q_{2}^{2}}-\left(q_{2}-q_{4}\right) \mathrm{i}-\left(q_{3}+\sqrt{q_{3}^{2}+q_{4}^{2}-q_{2}^{2}}\right) \mathrm{j}+\left(q_{2}-q_{4}\right) \mathrm{k}$.

3) If $q_{2}^{2}=q_{3}^{2}+q_{4}^{2}$, when $q_{2}=0$, then $q=q_{1}$, and

$$
x^{-1} q x=q_{1},
$$

in which $x=1$; When $q_{2} \neq 0$, then construct a nonsingular split quaternion $x$ such that

$$
x^{-1} q x=q_{1}+\mathrm{i}+\mathrm{j},
$$

in which $x=1+q_{3}+q_{4} \mathrm{i}+\left(q_{2}-1\right) \mathrm{k}$.

Remark 1 The statement above concludes that the set of v-quaternions is divided into four kinds of different equivalence classes. Moreover, if $q=q_{1}+q_{2} \mathrm{i}+q_{3} \mathrm{j}+q_{4} \mathrm{k}$ is a v-quaternion, then $q \in\left[q_{1}+\sqrt{q_{2}^{2}-v\left(q_{3}^{2}+q_{4}^{2}\right)} \mathrm{i}\right]$ if $q_{2}^{2}>v\left(q_{3}^{2}+q_{4}^{2}\right)$;

$q \in\left[q_{1}+\sqrt{q_{3}^{2}+q_{4}^{2}-\frac{1}{v} q_{2}^{2}} \mathrm{j}\right]$ if $q_{2}^{2}<v\left(q_{3}^{2}+q_{4}^{2}\right) ; q \in\left[q_{1}\right]$ if $q_{2}^{2}=v\left(q_{3}^{2}+q_{4}^{2}\right)$ and $q_{2}=0 ; q \in\left[q_{1}+\mathrm{i}+\frac{1}{\sqrt{v}} \mathrm{j}\right]$ if $q_{2}^{2}=v\left(q_{3}^{2}+q_{4}^{2}\right)$ and $q_{2} \neq 0$.

\section{Complex Representation of v-Quaternion Matrices}

For any v-quaternion matrix $A=A_{1}+A_{2} \mathrm{i}+A_{3} \mathrm{j}+A_{4} \mathrm{k}=\left(A_{1}+A_{2} \mathrm{i}\right)+\left(A_{3}+A_{4} \mathrm{i}\right)$ $\mathrm{j} \in \mathbf{H}_{v}^{m \times n}$, where $A_{1}, A_{2}, A_{3}, A_{4} \in \mathbf{R}^{m \times n}$, the complex representation of $A$ is defined to be

$$
A^{\sigma}=\left[\begin{array}{cc}
A_{1}+A_{2} \mathrm{i} & \epsilon \sqrt{v \epsilon}\left(A_{3}+A_{4} \mathrm{i}\right) \\
\sqrt{v \epsilon}\left(A_{3}-A_{4} \mathrm{i}\right) & A_{1}-A_{2} \mathrm{i}
\end{array}\right] \in \mathbf{C}^{2 m \times 2 n},
$$

in which $\epsilon=\left\{\begin{array}{l}1, v>0 \\ -1, v<0\end{array}\right.$. 
It is easy to prove that the mapping $A^{\sigma}$ is an isomorphism of ring $\mathbf{H}_{v}^{m \times n}$ to ring $\mathbf{C}^{2 m \times 2 n}$, and for $A, B \in \mathbf{H}_{v}^{\mathrm{m} \times n}, C \in \mathbf{H}_{v}^{n \times p}, a \in \mathbf{R}$, we have the following equalities.

$$
(A+B)^{\sigma}=A^{\sigma}+B^{\sigma},(A C)^{\sigma}=A^{\sigma} C^{\sigma},(a A)^{\sigma}=a A^{\sigma},
$$

and

$$
Q_{m}^{\mathrm{T}} A^{\sigma} Q_{n}=\overline{A^{\sigma}}, Q_{m}^{\mathrm{T}} \overline{A^{\sigma}} Q_{n}=A^{\sigma},
$$

in which $Q_{t}=\left[\begin{array}{cc}0 & \epsilon I_{t} \\ I_{t} & 0\end{array}\right], \epsilon=\left\{\begin{array}{l}1, v>0 \\ -1, v<0\end{array}, Q_{t}^{\mathrm{T}} Q_{t}=I_{2 t}, Q_{t}\right.$ is a unitary matrix, and clearly by (3.2) a v-quaternion matrix $A \in \mathbf{H}_{v}^{n \times n}$ is nonsingular if and only if $A^{\sigma}$ is nonsingular and $\left(A^{\sigma}\right)^{-1}=\left(A^{-1}\right)^{\sigma}$.

Remark 2 For two special cases of quaternion $(v=-1)$ and split quaternion $(v=1)$ matrices, clearly by (3.1) the complex representations are respectively to be

$$
A^{\sigma}=\left[\begin{array}{cc}
A_{1}+A_{2} \mathrm{i} & -A_{3}-A_{4} \mathrm{i} \\
A_{3}-A_{4} \mathrm{i} & A_{1}-A_{2} \mathrm{i}
\end{array}\right], A \in \mathbf{H}^{m \times n},
$$

and

$$
A^{\sigma}=\left[\begin{array}{ll}
A_{1}+A_{2} \mathrm{i} & A_{3}+A_{4} \mathrm{i} \\
A_{3}-A_{4} \mathrm{i} & A_{1}-A_{2} \mathrm{i}
\end{array}\right], A \in \mathbf{H}_{s}^{m \times n} .
$$

For any $A \in \mathbf{H}_{v}^{n \times n}$, if $\lambda \in \mathbf{C}$ is an eigenvalue of complex representation $A^{\sigma}$ of v-quaternion matrix $A$, i.e. there exists a nonzero complex vector $\beta=\left[\begin{array}{l}\beta_{1} \\ \beta_{2}\end{array}\right] \in \mathbf{C}^{2 n \times 1}, \beta_{1}, \beta_{2} \in \mathbf{C}^{n \times 1}$, such that $A^{\sigma} \beta=\beta \lambda$, then by (3.3) we have $A^{\sigma}\left(Q_{n} \bar{\beta}\right)=\left(Q_{n} \bar{\beta}\right) \bar{\lambda}$, and

$$
A^{\sigma} \beta=\beta \lambda \Leftrightarrow A^{\sigma}\left(\beta, Q_{n} \bar{\beta}\right)=\left(\beta, Q_{n} \bar{\beta}\right) \lambda^{\sigma} \Leftrightarrow A \alpha=\alpha \lambda,
$$

in which $\alpha=\beta_{1}+\frac{1}{\sqrt{v \epsilon}} \bar{\beta}_{2} \mathrm{j}$, and $\alpha^{\sigma}=\left(\beta, Q_{n} \bar{\beta}\right)$.

From the statement above we have the following results.

Proposition 3.1 Let $A \in \mathbf{H}_{v}^{n \times n}$, then

1) If $\lambda$ is a complex eigenvalue of complex representation $A^{\sigma}$, then $\bar{\lambda}$ is also a complex eigenvalue of complex representation $A^{\sigma}$. The imaginary eigenvalue of $A^{\sigma}$ appear in conjugate pairs, the number of real eigenvalues of $A^{\sigma}$ is even.

2) The v-quaternion matrix $A$ has at least a complex eigenvalue. Moreover, if $\lambda$ is a complex eigenvalue of $A^{\sigma}$ with $A^{\sigma} \beta=\beta \lambda$, let

$$
\alpha=\beta_{1}+\frac{1}{\sqrt{v \epsilon}} \bar{\beta}_{2} \mathrm{j}, \beta=\left[\begin{array}{c}
\beta_{1} \\
\beta_{2}
\end{array}\right] \in \mathbf{C}^{2 n \times 1}, \beta_{1}, \beta_{2} \in \mathbf{C}^{n \times 1},
$$

then $\lambda$ is a right eigenvalue of the v-quaternion matrix $A$, and $\alpha$ is a corresponding eigenvector related to right eigenvalue $\lambda$ with $A \alpha=\alpha \lambda$. 
Proposition 3.1 gives a relation between the right complex eigenvalues of complex representation $A^{\sigma}$ of a v-quaternion matrix $A$ and the right complex eigenvalues of the v-quaternion matrix $A$, and derives algebraic techniques for finding the right complex eigenvalues and corresponding eigenvectors of the $\mathrm{v}$-quaternion matrix $A$.

\section{Eigenvalues and Eigenvectors of v-Quaternion Matrices}

For $A \in \mathbf{H}_{v}^{n \times n}$, a nonzero v-quaternion $\lambda$, by [16], the complex representation matrix equation $A^{\sigma} X=X \lambda^{\sigma}$ has a nonzero solution $X \in \mathbf{C}^{2 n \times 2}$ if and only if $f_{A^{\sigma}}(x)$ and $f_{\lambda^{\sigma}}(x)$ are not relatively prime, i.e. $\left(f_{A^{\sigma}}(x), f_{\lambda^{\sigma}}(x)\right) \neq 1$, in other words, $f_{A^{\sigma}}\left(\lambda^{\sigma}\right)$ is a singular matrix.

Moreover, if there exists $X \in \mathbf{C}^{2 n \times 2}$ such that $A^{\sigma} X=X \lambda^{\sigma}$, by (3.3) we have

$$
A^{\sigma} X=X \lambda^{\sigma} \Leftrightarrow A^{\sigma}\left(Q_{n}^{\mathrm{T}} \bar{X} Q_{1}\right)=\left(Q_{n}^{\mathrm{T}} \bar{X} Q_{1}\right) \lambda^{\sigma} \Rightarrow A^{\sigma} Y=Y \lambda^{\sigma},
$$

in which $Y=\frac{1}{2}\left(X+Q_{n}^{\mathrm{T}} \bar{X} Q_{1}\right)$. Let

$$
X=\left[\begin{array}{ll}
X_{11} & X_{12} \\
X_{21} & X_{22}
\end{array}\right], X_{t s} \in \mathbf{C}^{n \times 1},
$$

then it is easy to get similarly that

$$
Y=\frac{1}{2}\left(X+Q_{n}^{\mathrm{T}} \bar{X} Q_{1}\right)=\left[\begin{array}{cc}
Y_{11} & Y_{12} \\
\epsilon \bar{Y}_{12} & \bar{Y}_{11}
\end{array}\right], Y_{t s} \in \mathbf{C}^{n \times 1},
$$

in which $Y_{11}=\frac{1}{2}\left(X_{11}+\bar{X}_{22}\right), Y_{12}=\frac{1}{2}\left(X_{12}+\epsilon \bar{X}_{21}\right), \epsilon=\left\{\begin{array}{l}1, v>0 \\ -1, v<0\end{array}\right.$.

By the fact that $\mathrm{j} \bar{Y}_{11}=Y_{11} \mathrm{j}, \mathrm{j} \bar{Y}_{12}=Y_{12} \mathrm{j}$, construct a v-quaternion $\alpha$, we can get the following equality by the direct calculation.

$$
\alpha=Y_{11}+\frac{\epsilon}{\sqrt{\epsilon V}} Y_{12} \mathrm{j}=\frac{1}{2}\left(I_{n}, \frac{1}{\sqrt{\epsilon V}} I_{n} \mathrm{j}\right) Y\left[\begin{array}{c}
1 \\
\frac{\epsilon}{\sqrt{\epsilon \mathcal{V}}} \mathrm{j}
\end{array}\right],
$$

then we have $\alpha^{\sigma}=Y$, and the v-quaternion $\lambda$ is a right eigenvalue of the v-quaternion matrix $A$ and $A \alpha=\alpha \lambda$.

The statements above imply following result.

Theorem 4.1 Let $A \in \mathbf{H}_{v}^{n \times n}, \lambda \in \mathbf{H}_{v}$. Then

1) The v-quaternion $\lambda$ is a right eigenvalue of the v-quaternion matrix $A$ if and only if $\left(f_{A^{\sigma}}(x), f_{\lambda^{\sigma}}(x)\right) \neq 1$, i.e. $f_{A^{\sigma}}\left(\lambda^{\sigma}\right)$ is a singular complex matrix;

2) The v-quaternion $\lambda$ is a right eigenvalue of $A$ if and only if complex representation matrix equation $A^{\sigma} Y=Y \lambda^{\sigma}$ has a nonzero solution $X \in \mathbf{C}^{2 n \times 2}$. In which case, if $X \in \mathbf{C}^{2 n \times 2}$ is a nonzero solution of $A^{\sigma} X=X \lambda^{\sigma}$, let

$$
\alpha=\frac{1}{4}\left(I_{n}, \frac{1}{\sqrt{\epsilon v}} I_{n} \mathrm{j}\right)\left(X+Q_{n}^{\mathrm{T}} \bar{X} Q_{1}\right)\left[\begin{array}{c}
1 \\
\frac{\epsilon}{\sqrt{\epsilon v}} \mathrm{j}
\end{array}\right]
$$

in which $Q_{t}=\left[\begin{array}{cc}0 & \epsilon I_{t} \\ I_{t} & 0\end{array}\right], \epsilon=\left\{\begin{array}{l}1, v>0 \\ -1, v<0\end{array}\right.$, then nonzero vector $\alpha \in \mathbf{H}_{v}^{n \times 1}$ satisfies 
$A \alpha=\alpha \lambda$. That is $\alpha$ is an eigenvector of the v-quaternion matrix $A$ related to the right eigenvalue $\lambda$.

This section researches the right eigenvalues and eigenvectors of a v-quaternion matrix with the help of the complex representation of the v-quaternion matrix, in this way, it makes the eigen-problem of the v-quaternion into a new topics, it is the matrix equation problem of complex representation. It also derives an algebraic technique to finding the right eigenvalues and corresponding eigenvectors of the v-quaternion.

\section{Algebraic Techniques for Eigenvalues and Eigenvectors}

For $A \in \mathbf{H}_{v}^{n \times n}$, let $V_{\lambda}=\left\{\beta \in \mathbf{C}^{2 n \times 1} \mid A^{\sigma} \beta=\beta \lambda\right\}$ be the characteristic subspace of complex eigenvalue $\lambda$. By Proposition 3.1, let all complex eigenvalues of complex representation $A^{\sigma}$ be as follows.

$$
\lambda_{1}, \bar{\lambda}_{1}, \cdots, \lambda_{t}, \bar{\lambda}_{t}, \mu_{1}, \mu_{2}, \cdots, \mu_{2 s},
$$

in which $\lambda_{\mu}$ are imaginary and $\mu_{v}$ are real, $1<\mu<t$ and $1<v<2 s$.

Case 1: For an imaginary eigenvalue $\lambda=\lambda_{u}$ of $A^{\sigma}$, let $A^{\sigma} \beta=\beta \lambda, \beta \neq 0$, by (3.3)

$$
A^{\sigma} \beta=\beta \lambda \Leftrightarrow A^{\sigma}\left(\beta, Q_{n} \bar{\beta}\right)=\left(\beta, Q_{n} \bar{\beta}\right) \lambda^{\sigma} .
$$

Then by (4.5) construct the nonzero vector $\alpha \in H_{v}^{n \times 1}$ satisfies $A \alpha=\alpha \lambda$. That is $\alpha$ is an eigenvector of the v-quaternion matrix $A$ related to the right eigenvalue $\lambda$.

Case 2: For a real eigenvalue $\lambda=\mu_{u}$ of $A^{\sigma}$, if there exist more than one different real eigenvalues, let $\lambda=\mu_{u}$ and $\mu=\mu_{v}$ be two different real eigenvalues, $A^{\sigma} \beta=\beta \lambda, A^{\sigma} \gamma=\gamma \mu$, then

$$
A^{\sigma}(\beta, \gamma)=(\beta, \gamma)\left[\begin{array}{ll}
\lambda & 0 \\
0 & \mu
\end{array}\right],
$$

therefore there exists non-negative real number $v>0$ such that

$$
\begin{aligned}
& A^{\sigma}(\beta, \gamma) P=(\beta, \gamma) P\left[\begin{array}{cc}
\frac{\lambda+\mu}{2} & \frac{\lambda-\mu}{2} \\
\frac{\lambda-\mu}{2} & \frac{\lambda+\mu}{2}
\end{array}\right] \Leftrightarrow A^{\sigma} Y=Y\left[\begin{array}{cc}
\frac{\lambda+\mu}{2} & \frac{\lambda-\mu}{2} \\
\frac{\lambda-\mu}{2} & \frac{\lambda+\mu}{2}
\end{array}\right] \\
& \Leftrightarrow A^{\sigma} Y=Y\left(\frac{\lambda+\mu}{2}+\frac{\lambda-\mu}{2 \sqrt{v}} \mathrm{j}\right)^{\sigma} \Leftrightarrow A^{\sigma} Y=Y \eta^{\sigma},
\end{aligned}
$$

in which $P=\left[\begin{array}{cc}1 & 1 \\ 1 & -1\end{array}\right], Y=(\beta, \gamma) P, \eta=\frac{\lambda+\mu}{2}+\frac{\lambda-\mu}{2 \sqrt{v}} \mathrm{j}$. Then by (4.5) construct the $\alpha \in \mathbf{H}_{v}^{n \times 1}$ satisfies $A \alpha=\alpha\left(\frac{\lambda+\mu}{2}+\frac{\lambda-\mu}{2 \sqrt{v}} \mathrm{j}\right)$. It is easy to prove that $\alpha$ is a nonzero vector by the fact that $\beta$ and $\gamma$ are linearly independent. That is $\alpha$ is an eigenvector of the v-quaternion matrix $A$ related to the right eigenvalue $\frac{\lambda+\mu}{2}+\frac{\lambda-\mu}{2 \sqrt{v}} \mathrm{j}$. 
Case 3: For real eigenvalue $\lambda$ of $A^{\sigma}$, if there exists only one real eigenvalue $\lambda$ and $\operatorname{dim} V_{\lambda}=1$, there exists a Jordan block $J(\lambda)$ of real eigenvalue $\lambda$ and a column full rank matrix $Y$ such that $A^{\sigma} Y=Y J(\lambda)$, then there exists two linearly independent vectors $\beta, \gamma$ such that $A^{\sigma}(\beta, \gamma)=(\beta, \gamma)\left[\begin{array}{ll}\lambda & 1 \\ 0 & \lambda\end{array}\right]$, and clearly

$$
A^{\sigma}(\beta, \gamma)=(\beta, \gamma)\left[\begin{array}{ll}
\lambda & 1 \\
0 & \lambda
\end{array}\right] \Leftrightarrow A^{\sigma} Y=Y\left[\begin{array}{cc}
\lambda+\mathrm{i} & 1 \\
1 & \lambda-\mathrm{i}
\end{array}\right],
$$

and clear that $\left[\begin{array}{ll}\lambda & 1 \\ 0 & \lambda\end{array}\right]=P\left[\begin{array}{cc}\lambda+\mathrm{i} & 1 \\ 1 & \lambda-\mathrm{i}\end{array}\right] P^{-1}$, in which $P=\left[\begin{array}{ll}1 & 0 \\ \mathrm{i} & 1\end{array}\right]$.

Therefore there only exists non-negative real number $v>0$ such that

$$
A^{\sigma} Y=Y\left(\lambda+\mathrm{i}+\frac{1}{\sqrt{v}} \mathrm{j}\right) \Leftrightarrow A^{\sigma}(\beta, \gamma) P=(\beta, \gamma) P\left[\begin{array}{cc}
\lambda+\mathrm{i} & 1 \\
1 & \lambda-\mathrm{i}
\end{array}\right],
$$

in which $Y=(\beta, \gamma) P=(\beta, \gamma)\left[\begin{array}{ll}1 & 0 \\ \mathrm{i} & 1\end{array}\right]=(\beta+\gamma \mathrm{i}, \gamma)$. Then by (4.5) construct the $\alpha \in \mathbf{H}_{v}^{n \times 1}$ satisfies $A \alpha=\alpha\left(\lambda+\mathrm{i}+\frac{1}{\sqrt{v}} \mathrm{j}\right)$. It is easy to prove that $\alpha$ is a nonzero vector by the fact that $\beta$ and $\gamma$ are linearly independent. That is $\alpha$ is an eigenvector of the v-quaternion matrix $A$ related to the right eigenvalue $\lambda+\mathrm{i}+\frac{1}{\sqrt{v}} \mathrm{j}$.

Case 4: For real eigenvalue $\lambda$ of $A^{\sigma}$, If there exists only one real eigenvalue $\lambda$ of $A^{\sigma}$, and $\operatorname{dim} V_{\lambda} \geq 2$, there exist two linearly independent vectors $\beta, \gamma \in V_{\lambda}$ such that $A^{\sigma} \beta=\beta \lambda, A^{\sigma} \gamma=\gamma \lambda$, then

$$
A^{\sigma} \beta=\beta \lambda, A^{\sigma} \gamma=\gamma \lambda \Leftrightarrow A^{\sigma}(\beta, \gamma)=(\beta, \gamma)\left[\begin{array}{ll}
\lambda & 0 \\
0 & \lambda
\end{array}\right] \Leftrightarrow A^{\sigma} Y=Y \lambda^{\sigma},
$$

in which $Y=(\beta, \gamma)$. Then by (4.5) construct the $\alpha \in \mathbf{H}_{v}^{n \times 1}$ satisfies $A \alpha=\alpha \lambda$, and $\alpha$ is a nonzero vector by the fact that $\beta$ and $\gamma$ are linearly independent. That is $\alpha$ is an eigenvector of the v-quaternion matrix $A$ related to the right eigenvalue $\lambda$.

For $A \in \mathbf{H}_{v}^{n \times n}$, if a v-quaternion $\lambda$ is a right eigenvalue of $A$, and $A \alpha=\alpha \lambda$, then for any nonsingular v-quaternion $p$, we have $A(\alpha p)=(\alpha p)\left(p^{-1} \lambda p\right)$, i.e. $p^{-1} \lambda p$ is a right eigenvalue of $A$ with an eigenvector $\alpha p$.

\section{Algorithm and Example Algorithm}

Let $A \in \mathbf{H}_{v}^{n \times n}$. Then an algorithm for right v-quaternion eigenvalues and eigenvectors of the v-quaternion matrix is given as follows.

1) Compute the complex representation $A^{\sigma}$ of $A$ by (3.1).

2) Compute all eigenvalues of $A^{\sigma}$. Let all the eigenvalues of $A^{\sigma}$ be as follows.

$$
\lambda_{1}, \bar{\lambda}_{1}, \cdots, \lambda_{t}, \bar{\lambda}_{t}, \mu_{1}, \mu_{2}, \cdots, \mu_{2 s}
$$


in which $\lambda_{\mu}$ are imaginary and $\mu_{v}$ are real, $1<\mu<t$ and $1<v<2 s$.

3) Choose one of each pair of conjugate complex eigenvalues and all the real eigenvalues of $A^{\sigma}$. Then can get the related eigenvalues of $A$.

4) Search the eigenvectors related to eigenvalues of the $A^{\sigma}$ in step 3.

5) If $\lambda$ is an eigenvalue of the v-quaternion matrix $A$, then it is easy to get a $Y \in \mathbf{C}^{2 n \times 2}$ such that $A^{\sigma} Y=Y \lambda^{\sigma}$ by finding a solution of complex matrix equation $A^{\sigma} X=X \lambda^{\sigma}$. Let

$$
\alpha=\frac{1}{4}\left(I_{n}, \frac{1}{\sqrt{\epsilon \mathcal{V}}} I_{n} \mathrm{j}\right)\left(Y+Q_{n}^{\mathrm{T}} \bar{Y} Q_{1}\right)\left[\begin{array}{c}
1 \\
\frac{\epsilon}{\sqrt{\epsilon \mathcal{V}}}
\end{array}\right],
$$

then nonsingular vector $\alpha \in \mathbf{H}_{v}^{n \times 1}$ satisfies $A \alpha=\alpha \lambda$. That is $\alpha$ is an eigenvector of the v-quaternion matrix $A$ related to the right eigenvalue $\lambda$.

6) Find out the equivalence classes of all the eigenvalues, find the related eigenvectors of v-quaternion $A$.

Example Let

$$
A=\left[\begin{array}{cc}
2+\mathrm{i}-2 \mathrm{j} & 2-4 \mathrm{j}-2 \mathrm{k} \\
-\mathrm{i}+\mathrm{j} & 1-\mathrm{i}+2 \mathrm{j}+\mathrm{k}
\end{array}\right]
$$

The complex representation of the v-quaternion matrix $A$ is as follows

$$
A^{\sigma}=\left[\begin{array}{cccc}
2+\mathrm{i} & 2 & -2 \epsilon \sqrt{v \epsilon} & -2 \epsilon(2 \sqrt{v \epsilon}+\sqrt{-v \epsilon}) \\
-\mathrm{i} & 1-\mathrm{i} & \epsilon \sqrt{v \epsilon} & \epsilon(2 \sqrt{v \epsilon}+\sqrt{-v \epsilon}) \\
-2 \sqrt{v \epsilon} & -4 \sqrt{v \epsilon}+2 \sqrt{-v \epsilon} & 2-\mathrm{i} & 2 \\
\sqrt{v \epsilon} & 2 \sqrt{v \epsilon}-\sqrt{-v \epsilon} & \mathrm{i} & 1+\mathrm{i}
\end{array}\right]
$$

in which $\epsilon=\left\{\begin{array}{l}1, v>0 \\ -1, v<0\end{array}\right.$.

The following two examples are special cases of the v-quaternion.

Case 1: For quaternion case with $(v=-1)$. It is easy to know $A^{\sigma}$ by (3.4a),

$$
A^{\sigma}=\left[\begin{array}{cccc}
2+\mathrm{i} & 2 & 2 & 4+2 \mathrm{i} \\
-\mathrm{i} & 1-\mathrm{i} & -1 & -2-\mathrm{i} \\
-2 & -4+2 \mathrm{i} & 2-\mathrm{i} & 2 \\
1 & 2-\mathrm{i} & \mathrm{i} & 1+\mathrm{i}
\end{array}\right]
$$

by the direct calculation get the eigenvalues of $A^{\sigma}$ are $\lambda_{\mu}=2+\mathrm{i}, \bar{\lambda}_{\mu}=2-\mathrm{i}$, $\lambda_{\eta}=1+\sqrt{2} \mathrm{i}, \bar{\lambda}_{\eta}=1-\sqrt{2} \mathrm{i}$.

Choose two eigenvalues $\lambda_{\mu}=2+\mathrm{i}, \lambda_{\eta}=1+\sqrt{2} \mathrm{i}$ of two pairs of conjugate eigenvalues.

For the eigenvalue $\lambda_{\mu}=2+\mathrm{i}$. It is easy to get that $A^{\sigma} \beta=\beta \lambda$, in which $\beta=(2,-1,-1-\mathrm{i}, 1)^{\mathrm{T}}$. By (4.5), let

$$
\alpha=\frac{1}{4}\left(I_{2}, I_{2} \mathrm{j}\right)\left(\left(\beta, Q_{2} \bar{\beta}\right)+Q_{2}^{\mathrm{T}}\left(\bar{\beta}, Q_{2} \beta\right) Q_{1}\right)\left[\begin{array}{c}
1 \\
-\mathrm{j}
\end{array}\right]=\left[\begin{array}{c}
2-\mathrm{j}-\mathrm{k} \\
-1+\mathrm{j}
\end{array}\right],
$$

then $A \alpha=\alpha \lambda_{\mu}, \alpha$ is an eigenvector related to $\lambda_{\mu}=2+\mathrm{i}$. Moreover, for any 
nonsingular quaternion $p$, quaternion $p^{-1} \lambda_{\mu} p$ is a right eigenvalue of $A$ with an eigenvector $\alpha p$.

In the same way, for the eigenvalue $\lambda_{\eta}=1+\sqrt{2} \mathrm{i}$. It is easy to get that $A^{\sigma} \beta=\beta \lambda_{\eta}$, in which $\beta=(2+2 \sqrt{2},-1-\sqrt{2},-2,1)^{\mathrm{T}}$. By (4.5), let

$$
\alpha=\frac{1}{4}\left(I_{2}, I_{2} \mathrm{j}\right)\left(\left(\beta, Q_{2} \bar{\beta}\right)+Q_{2}^{\mathrm{T}}\left(\bar{\beta}, Q_{2} \beta\right) Q_{1}\right)\left[\begin{array}{c}
1 \\
-\mathrm{j}
\end{array}\right]=\left[\begin{array}{c}
2(1+\sqrt{2}-\mathrm{j}) \\
-1-\sqrt{2}+\mathrm{j}
\end{array}\right],
$$

then $A \alpha=\alpha \lambda_{\eta}, \alpha$ is an eigenvector related to $\lambda_{\eta}=1+\sqrt{2} \mathrm{i}$. Moreover, for any nonsingular quaternion $p$, quaternion $p^{-1} \lambda_{\eta} p$ is a right eigenvalue of $A$ with an eigenvector $\alpha p$.

Case 2: For split quaternion case with $(v=1)$. It is easy to know $A^{\sigma}$ by (3.4b),

$$
A^{\sigma}=\left[\begin{array}{cccc}
2+\mathrm{i} & 2 & -2 & -4-2 \mathrm{i} \\
-\mathrm{i} & 1-\mathrm{i} & 1 & 2+\mathrm{i} \\
-2 & -4+2 \mathrm{i} & 2-\mathrm{i} & 2 \\
1 & 2-\mathrm{i} & \mathrm{i} & 1+\mathrm{i}
\end{array}\right],
$$

it is easy to get the eigenvalues of $A^{\sigma}$ are $\lambda=2+\mathrm{i}, \bar{\lambda}=2-\mathrm{i}, \mu=1$, and $\operatorname{dim} V_{\mu}=1$.

For two conjugate imaginary eigenvalues $\lambda=2+\mathrm{i}, \bar{\lambda}=2-\mathrm{i}$, choose an eigenvalue $\lambda=2+\mathrm{i}$ of a pairs of conjugate eigenvalues. For the only one real eigenvalue $\mu=1$ with $\operatorname{dim} V_{\mu}=1$, by (5.6) get an eigenvalue $\lambda=1+\mathrm{i}+\mathrm{j}$ of $A$.

For the principal eigenvalue $\lambda=2+\mathrm{i}$, It is easy to get that $A^{\sigma} \beta=\beta \lambda$, in which $\beta=(2,-1,-1,0)^{\mathrm{T}}$. By $(4.5)$, let

$$
\alpha=\frac{1}{4}\left(I_{2}, I_{2} \mathrm{j}\right)\left(\left(\beta, Q_{2} \bar{\beta}\right)+Q_{2}^{\mathrm{T}}\left(\bar{\beta}, Q_{2} \beta\right) Q_{1}\right)\left[\begin{array}{l}
1 \\
\mathrm{j}
\end{array}\right]=\left[\begin{array}{c}
2-\mathrm{j} \\
-1
\end{array}\right],
$$

then $A \alpha=\alpha \lambda, \alpha$ is an eigenvector related to $\lambda=2+\mathrm{i}$. Moreover, for any nonsingular split quaternion $p$, split quaternion $p^{-1} \lambda p$ is a right eigenvalue of $A$ with an eigenvector $\alpha p$.

For the principal eigenvalue $\mu=1$ with $\operatorname{dim} V_{\mu}=1$. It is easy to get two linearly independent vectors $\beta, \gamma$ such that

$$
A^{\sigma}(\beta, \gamma)=(\beta, \gamma)\left[\begin{array}{ll}
\mu & 1 \\
0 & \mu
\end{array}\right],
$$

in which $\beta=(2,-1,-2,1)^{\mathrm{T}}, \quad \gamma=\left(-\mathrm{i}, \frac{\mathrm{i}}{2},-\mathrm{i}, \frac{\mathrm{i}}{2}\right)^{\mathrm{T}}$. By (4.5), let

$$
\alpha=\frac{1}{4}\left(I_{2}, I_{2} \mathrm{j}\right)\left((\beta+\gamma \mathrm{i}, \gamma)+Q_{2}^{\mathrm{T}}(\bar{\beta}-\bar{\gamma} \mathrm{i}, \bar{\gamma}) Q_{1}\right)\left[\begin{array}{l}
1 \\
\mathrm{j}
\end{array}\right]=\left[\begin{array}{c}
3+\mathrm{i}-\mathrm{j}-\mathrm{k} \\
-\frac{3}{2}-\frac{1}{2} \mathrm{i}+\frac{1}{2} \mathrm{j}+\frac{1}{2} \mathrm{k}
\end{array}\right],
$$

then $A \alpha=\alpha \lambda, \alpha$ is an eigenvector related to $\lambda=\mu+\mathrm{i}+\mathrm{j}=1+\mathrm{i}+\mathrm{j}$. Moreover, for any nonsingular split quaternion $p$, split quaternion $p^{-1} \lambda p$ is a right eigenvalue of $A$ with an eigenvector $\alpha p$. 


\section{Conclusion}

This paper gives the concept of the v-quaternions, and studies eigenvalues and eigenvectors of the v-quaternion matrices by means of the complex representation of the v-quaternion matrices, and derives an algebraic technique to find the eigenvalues and eigenvectors of v-quaternion matrices. This paper also gives a unification of algebraic techniques for eigenvalues and eigenvectors in quaternionic and split quaternionic mechanics.

\section{Acknowledgements}

We thank the Editor and the referee for their comments. Research of T. Jiang is funded by the National Natural Science Foundation of China (11771188) and Shandong Natural Science Foundation (ZR201709250116). This support is greatly appreciated.

\section{Conflicts of Interest}

The authors declare no conflicts of interest regarding the publication of this paper.

\section{References}

[1] Hamilton, W.R. (1843) On a New Species of Imaginary Quantities Connected with a Theory of Quaternions. Proceedings of the Royal Irish Academy, 2, 424-434.

[2] Cockle, J. (1849) On Systems of Algebra Involving More than One Imaginary; and on Equations of the Fifth Degree. Philosophical Magazine, 35, 434-437.

[3] Adler, S.L. (1995) Quaternionic Quantum Mechanics and Quantum Fields. Oxford University Press, New York.

[4] Pereira, R., Rocha, P. and Vettori, P. (2005) Algebraic Tools for the Study of Quaternionic Behavioral Systems. Linear Algebra and Its Applications, 400, 121-140. https://doi.org/10.1016/j.laa.2005.01.008

[5] Rodman, L. (2014) Topics in Quaternion Linear Algebra. Princeton University Press, Princeton, NJ. https://doi.org/10.23943/princeton/9780691161853.001.0001

[6] Brody, D.C. and Graefe, E.M. (2011) On Complexified Mechanics and Coquaternions. Journal of Physics A: Mathematical and Theoretical, 44, Article ID: 072001. https://doi.org/10.1088/1751-8113/44/7/072001

[7] Kula, L. and Yayli, Y. (2007) Split Quaternions and Rotations in Semi Euclidean Space. Journal of the Korean Mathematical Society, 44, 1313-1327. https://doi.org/10.4134/JKMS.2007.44.6.1313

[8] Özdemir, M. and Ergin, A.A. (2005) Some Geometric Applications of Timelike Quaternions. International Conference of The Jangjeon Mathematical Society, 6, 108-115.

[9] Özdemir, M. and Ergin, A.A. (2006) Rotations with Unit Timelike Quaternions in Minkowski 3-Space. Journal of Geometry and Physic, 56, 322-336. https://doi.org/10.1016/j.geomphys.2005.02.004

[10] Jiang, T. (2004) An Algorithm for Eigenvalues and Eigenvectors of Quaternion Matrices in Quaternionic Quantum Mechanics. Journal of Mathematical Physics, 45, 3334-3338. https://doi.org/10.1063/1.1769106

[11] Baker, A. (1999) Right Eigenvalues for Quaternionic Matrices: A Topological Ap- 
proach. Linear Algebra and its Applications, 286, 303-309.

https://doi.org/10.1016/S0024-3795(98)10181-7

[12] Jiang, T., Zhang, Z. and Jiang, Z. (2018) Algebraic Techniques for Eigenvalues and Eigenvectors of a Split Quaternion Matrix in Split Quaternionic Mechanics. Computer Physics Communications, 229, 1-7. https://doi.org/10.1016/j.cpc.2018.03.021

[13] Erdoğdu, M. and Özdemir, M. (2013) On Eigenvalues of Split Quaternion Matrices. Advances in Applied Clifford Algebras, 23, 615-623. https://doi.org/10.1007/s00006-013-0391-7

[14] Brody, D.C. and Graefe, E.M. (2011) Coquaternionic Quantum Dynamics for Two-Level Systems. Acta Polytechnica Hungarica, 51, 14-20.

[15] Bender, C.M. (2007) Making Sense of Non-Hermitian Hamiltonians. Reports on Progress in Physics, 70, 947-1018. https://doi.org/10.1088/0034-4885/70/6/R03

[16] Lancaster, P. and Tismenersky, M. (1985) The Theory of Matrices with Applications. Academic Press, New York. 